STRUCTURAL BIOLOGY

ISSN 2059-7983

Keywords: atomic resolution; structural biology; response.

\section{Responses to 'Atomic resolution': a badly abused term in structural biology}

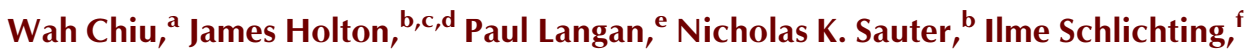 \\ Tom Terwilliger, ${ }^{\mathrm{g}}$ Jennifer L Martin, ${ }^{\mathrm{h}_{*}}$ Randy J. Read ${ }^{\mathrm{i} *}$ and Soichi Wakatsuki ${ }^{\mathrm{i} *}$
}

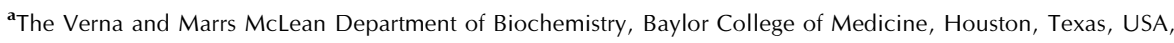
${ }^{\mathbf{b}}$ Molecular Biophysics \& Integrated Bioimaging Division, Lawrence Berkeley National Laboratory, Berkeley, USA, 'Stanford Synchrotron Radiation Lightsource, SLAC National Accelerator Laboratory, Menlo Park, USA, dDepartment of Biochemistry and Biophysics, University of California, San Francisco, San Francisco, USA, ' Oak Ridge National Laboratory, Oak Ridge, Tennessee, USA, 'Department of Biomolecular Mechanisms, Max Planck Institute for Medical Research, Heidelberg, Germany, ${ }^{\mathbf{g}}$ Bioscience Division, Los Alamos National Laboratory, Los Alamos, New Mexico, USA, ${ }^{\mathbf{h}}$ The Eskitis Drug Discovery Institute, N27, Griffith University, Nathan, Australia, 'Cambridge Institute for Medical Research, Department of Haematology, University of Cambridge, Wellcome Trust/MRC Building, Hills Road, Cambridge, United Kingdom, and 'Photon Science, SLAC and Structural Biology, School of Medicine, Stanford University, Menlo Park, USA. *Correspondence e-mail: eskitisdirector@griffith.edu.au, rjr27@cam.ac.uk, soichi.wakatsuki@stanford.edu

The Editors of Acta Cryst. $D$ have received a Letter to the Editor from Alex Wlodawer and Zbigniew Dauter (Wlodawer \& Dauter, 2017) concerning the use of the term atomic resolution. As this is an important topic, a number of representatives from the structural biology community were asked to respond to the Letter and their comments are given here.

The IUCr Commission on Biological Macromolecules (chair Tom Terwilliger) responded as follows:

The IUCr Commission on Biological Macromolecules endorses the concepts in the Letter to the Editor by Dauter and Wlodawer and in particular supports the idea of developing community-agreed working definitions of 'atomic resolution' and 'nearatomic resolution'. The Commission notes that although there are limitations in the definition of 'resolution' itself these limitations should not prevent the X-ray, neutron, XFEL, cryo-EM and other communities from coming to a consensus on the use of the terms 'atomic resolution' and 'near-atomic resolution', as suggested in the letter.

Nicholas K. Sauter, a Senior Scientist at the Lawrence Berkeley National Laboratory responded as follows:

The resolution limit as a concept itself is especially susceptible to misuse. In the last few years the Karplus \& Diederichs research on information content (leading to the introduction of $\mathrm{CC}_{1 / 2}$ ) has shown that it is advantageous to include very weak and uncertain data that is near the resolution limit (Karplus \& Diederichs, 2015). But the simple fact of including that data does not mean that the map resolution is really at that limit. The situation is especially applicable to XFEL experiments where weak data are routinely included at the limit of significance (Sauter, 2015). This all suggests it might be best to refocus on the concept of 'optical resolution', that is, can a map actually resolve the difference between two atoms? There are two excellent papers on this subject from Drs Dauter (Luo et al., 2014) and Urzhumtsev (Urzhumtseva et al., 2013). So to summarize, the Letter to the Editor emphasizes the distinction between 'atomic resolution' and 'near-atomic resolution'. I think that even the 'resolution' part of the expression can be misused: just because we include data to a certain limit doesn't mean it has that information content.

Wah Chiu, from Baylor College of Medicine and part of the biological cryo-EM community, had the following to say,

I am sympathetic to the concern expressed by these authors about the use of the term 'atomic resolution' and 'near atomic resolution'. I agree that we should standardize this vocabulary so that they have a defined meaning to the scientific community. 
James Holton from LBNL, SLAC and the University of California, San Francisco said

I agree that the term 'resolution' needs some clarity. First of all the use of the words 'high', and 'low' or 'above' and 'below' are themselves ambiguous. Is 2 higher than 3 ? Personally, I try to use 'inner' and 'outer' wherever I can, or at least 'high-angle' or 'low-angle'. That is at least unambiguous. And yes, if we can't even agree on a sign convention we are definitely in trouble on magnitude. That said, I think the small-molecule definition for 'atomic resolution' is a bit draconian when it comes to macromolecular work. Is there any question that a macromolecular structure at $3.0 \AA$ can locate atoms? If not, then why do we have a PDB? The $1.2 \AA$ 'Sheldrick criterion' is actually an operational one. It is the resolution at which SHELX works. I have no doubt that if SHELX could solve a structure by direct methods at $2.5 \AA$ then that would be considered 'atomic resolution'. So what is a good criterion? I would hearken back to the optical definition of 'resolution', which is the minimum distance between two points that can be 'resolved'. This is actually much more closely related to the atomic $B$ factors than anything else, because two Gaussians a certain distance apart loose the 'dip' between the two of them. That's the point where the two atoms start to be 'resolved'. This distance is 0.8493 the full-width at half-maximum of the two Gaussians, leading to the expression:

$$
\text { resolution }=0.8493\left[B \log (2) / \pi^{2}+0.816^{2}\right]^{1 / 2} \text {. }
$$

The 0.816 value is the average full-width-half-maximum of a protein atom with $B$ factor set to zero. This gets convoluted with the atomic $B$ factor, which quadratureadds a FWHM of $\sim(B / 14.2)^{1 / 2}$. But this point is already moot, because you can always sharpen the $B$ factors. The trick then, is the signal-to-noise ratio, and you want to define a point where the difference between being 'resolved' and not for a map sharpened all the way ( $E$ values) becomes indistinguishable because of noise. Lang et al. (2014) laid the groundwork for this with END/ RAPID maps. The extension would perhaps be called sharp-END/RAPID maps. What I expect will happen is that the high-angle noise will dominate and the signal-tonoise ratio of the sharp-END electron density will be essentially equal to that of the normalized structure factors (via Parseval's theorem) and the 'resolution' will be a $d$-spacing a bit beyond where $I / \sigma(I)$ drops to zero. The relationship between 'resolution' and a particular atomic $B$ factor is an important one I think, because it highlights how different parts of the map can be at different 'resolution' (Gicovazzo \& Mazzone, 2012; Lattman, 1990). Hopefully, this is a reminder that even achieving 'atomic resolution' is never a blank check, allowing you to make any conclusions you want about your structure. Real scientific conclusions always come from either a clear qualitative difference or a 'statistically significant' differ- ence from a control. That 'control' can be the long-term experience of a field in the former case, but not in the latter.

Ilme Schlichting, Director at the Max Planck Institute for Medical Research, Heidelberg and one of the Main Editors of the Journal of Synchrotron Radiation, responded in the following way:

I wholeheartedly support the Letter to the Editor by Zbigniew Dauter and Alexander Wlodawer. It is also very timely as shown for example by a recent discussion on the meaning of 'high resolution' on the CCP4 bulletin board. If taken literally, the meaning of 'atomic resolution' should be clear, as pointed out in the letter: the ability to see separate atoms. However, increasingly, it seems to be used to express that the resolution of the structure is 'high' in comparison to previous studies. This applies in particular to rapidly developing methods such as single particle reconstruction by cryo-EM and serial femtosecond crystallography (SFX) at XFELs. In the very least, this inflation of the 'atomic resolution' term is confusing since it loses its meaning. In general, I do not see the need to label the resolution with a descriptive adjective. A number should be sufficient. However, different methods use different criteria to assess not only the resolution but also the quality of the data (completeness, $\mathrm{CC}_{1 / 2}$, number of NOEs/residue, Fourier shell coefficients etc.). Both are important because both determine what one can ultimately resolve. It is also here that I see a need for consensus of how to make sure (and demonstrate) that the data quality also supports the resolution claim. For example, when demonstrating for the first time that high resolution structures can be obtained by SFX at XFELs we knew that our resolution was indeed better than $2 \AA$ because we could resolve the two $\mathrm{S}$ atoms in disulfides (S-S distance is $2 \AA$ ).

Paul Langan, Associate Laboratory Director for Neutron Sciences at Oak Ridge National Laboratory, gives the following point of view:

In the article, Wlodawer \& Dauter postulate that all structural communities adopt standard definitions of terms such as 'atomic resolution' and 'near-atomic resolution'. By doing so readers of structural papers may get a realistic impression of the accuracy of structures based on the reported resolution of the primary experimental data. Relating the accuracy of structures to the reported resolution of the primary data would be very helpful to readers. However, the challenge is that the relationship between the reported resolution of the primary data, the resolving power of density maps calculated from them and the accuracy of a structure determined from them differ across techniques used for structure determination and cannot always be usefully represented by simple criteria or data limits.

To illustrate this challenge, consider the techniques of $\mathrm{X}$-ray and neutron crystallography referred to in the 
article. Whereas X-ray crystallography remains the primary technique for determining the structure of biological macromolecules, neutron crystallography is typically used not to determine new structures, but rather to answer outstanding scientific questions by locating the presence or absence of hydrogen $(\mathrm{H})$ atoms or ions after the structure has been determined using X-rays (Chen \& Langan, 2013). Electron-density maps calculated from data collected to a value of $d_{\min }<1.2 \AA$ should have sufficient resolving power to distinguish the presence of $\mathrm{H}$ atoms (Sheldrick, 1990), and yet it is often not possible to image those atoms (Howard et al., 2004). This can be due to several factors including high thermal displacements, data completeness, anisotropy of the distribution of reflections in reciprocal space, or the weak X-ray scattering power of $\mathrm{H}$. The important point is that $d_{\min }$ on its own is not always sufficient to predict the resolving power of an electron-density map. Further, density maps used in the later stages of structure determination are biased by the use of model phases, and their resolving power is therefore dependent on the accuracy of the model. These limitations in using $d_{\min }$ on its own to predict the resolving power of density maps are similar for neutron crystallography, of course.

On the other hand, it continues to be demonstrated that neutron crystallography can be used to directly resolve a single, functionally important, $\mathrm{H}$ atom, in or around a biological macromolecule even with data collected at room temperature to a value of $d_{\min }>2 \AA$ (e.g. Banco et al., 2016; Gerlits et al., 2016; Knihtila et al., 2015; Kwon et al., 2016; Langan et al., 2016). The important difference is that X-ray and neutron data sets collected to the same $d_{\text {min }}$ can be used to calculate density maps that have different information content. The greater information content of nuclear density maps is due to the neutron scattering length of $\mathrm{H}$, which appears as a negative trough in nuclear density maps (most other atoms found in biological molecules such as $\mathrm{C}, \mathrm{N}, \mathrm{O}, \mathrm{S}$ and $\mathrm{P}$ appear as positive peaks) (Afonine et al., 2010). The ability to resolve $\mathrm{H}$ atoms or ions is enhanced further by the substitution of $\mathrm{H}$ atoms by deuterium (D) in a molecule (called deuteration) (Vandavasi et al., 2016). D appears as a positive peak twice the magnitude of $\mathrm{H}$ in nuclear density maps with a neutron scattering length equal to C. Further improvement in the accuracy of the positions of atoms in a neutron crystal structure can be obtained by refining their coordinates using both X-ray and neutron data sets in joint refinement procedures. Interestingly, the positions of atoms in an X-ray crystal structure refined from data collected to a certain value of $d_{\min }$ can be significantly improved by including neutron data collected to a much larger value of $d_{\text {min }}$ (Adams et al., 2009).

Wlodawer and Dauter have identified a need for agreement on the use of standard terms at a time when researchers are increasingly using multiple techniques to determine and model the structure and dynamics of biological macromolecules. This is an important topic, but also a complicated one. The challenges in defining data resolution and its relationship to the resolving power of the density maps discussed above for X-ray and neutron crystallography are greatly increased when broadened to include cryo-EM and NMR, in which the resolution of data has less to do with the resolving power of maps. However, this should not be a deterrent to continuing to explore and develop new techniques to determine and model biological molecules and also new innovative approaches to combining information from multiple techniques to amplify the completeness and the accuracy of those models.

Paul Langan thanks Paul Adams, Pavel Afonine, Leighton Coates and Andrey Kovalevsky for helpful discussions.

All the authors here are thanked for taking time to consider this subject and for their contributions. We would encourage representative leaders of the different technologies to work with the IUCr Commissions and write a perspective paper on this topic, for a future journal issue.

\section{References}

Adams, P. D., Mustyakimov, M., Afonine, P. V. \& Langan, P. (2009). Acta Cryst. D65, 567-573.

Afonine, P. V., Mustyakimov, M., Grosse-Kunstleve, R. W., Moriarty, N. W., Langan, P. \& Adams, P. D. (2010). Acta Cryst. D66, 11531163.

Banco, M. T., Mishra, V., Ostermann, A., Schrader, T. E., Evans, G. B., Kovalevsky, A. \& Ronning, D. R. (2016). Proc. Natl Acad. Sci. USA, 113, 13756-13761.

Chen, J. \& Langan, P. (2013). Phys. Chem. Chem. Phys. 15, $13705-$ 13712.

Gerlits, O., Wymore, T., Das, A., Shen, C., Parks, J. M., Smith, J. C., Weiss, K., Keen, D. A., Blakeley, M. P., Louis, J. M., Langan, P., Weber, I. \& Kovalevsky, A. (2016). Angew. Chem. Int. Ed. 55, 49244927.

Giacovazzo, C. \& Mazzone, A. (2012). Acta Cryst. A68, 464-469.

Howard, E. I., Sanishvili, R., Cachau, R. E., Mitschler, A., Chevrier, B., Barth, P., Lamour, V., Van Zandt, M., Sibley, E., Bon, C., Moras, D., Schneider, T. R., Joachimiak, A. \& Podjarny, A. (2004). Proteins, 55, 792-804.

Karplus, P. A. \& Diederichs, K. (2015). Curr. Opin. Struct. Biol. 34, 60-68.

Knihtila, R., Holzapfel, G., Weiss, K., Meilleur, F. \& Mattos, C. (2015). J. Biol. Chem. 290, 31025-31036.

Kwon, H., Basran, J., Casadei, C. M., Fielding, A. J., Schrader, IT. E., Ostermann, A., Devos, J. M., Aller, P., Blakeley, M. P., Moody, P. C. \& Raven, E. L. (2016). Nat. Commun. 7, 13445-13445.

Lang, P. T., Holton, J. M., Fraser, J. S \& Alber, T. (2014). Proc. Natl Acad. Sci. USA, 111, 237-242.

Langan, P. S., Close, D. W., Coates, L., Rocha, R. C., Ghosh, K., Kiss, C., Waldo, G., Freyer, J., Kovalevsky, A. \& Bradbury, A. R. (2016). J. Mol. Biol. 428, 1776-1789.

Lattman, E. E. (1990). Acta Cryst. A46, 628-630.

Luo, Z., Rajashandar, K. \& Dauter, Z. (2014). Acta Cryst. D70, $253-$ 260.

Sauter, N. K. (2015). J. Synchrotron Rad. 22, 239-248.

Sheldrick, G. M. (1990). Acta Cryst. A46, 467-473.

Urzhumtseva, L., Klaholz, B. \& Urzhumtsev, A. (2013). Acta Cryst. D69, 1921-1934.

Vandavasi, V. G., Weiss, K. L., Cooper, J. B., Erskine, P. T., Tomanicek, S. J., Ostermann, A., Schrader, T. E., Ginell, S. L. \& Coates, L. (2016). J. Med. Chem. 59, 474-479.

Wlodawer, A. \& Dauter, Z. (2017). Acta Cryst. D73, 379-380. 